\title{
4-Phosphothiophen-2-yl alanine: a new 5-membered analogue of phosphotyrosine $\dagger$
}

Cite this: Chem. Commun., 2014, 50, 9343

Received 6th May 2014 , Accepted 25th June 2014

DOI: $10.1039 / c 4 c c 03393 k$

www.rsc.org/chemcomm

\author{
Matthew Lilley, ${ }^{\text {ab }}$ Bezaleel Mambwe, ${ }^{b}$ Richard F. W. Jackson ${ }^{\star a}$ and \\ Richmond Muimo*b
}

Polyclonal antibodies raised against 4-phosphothiophen-2-yl alanine $2 a$, a novel five-membered ring analogue of phosphotyrosine, showed high selectivity for phosphotyrosine and no cross-reactivity with other phosphorylated amino acids. Western blots showed that the polyclonal was similarly effective, but different in selectivity, to a commercially available monoclonal antibody.

Protein tyrosine phosphatases (PTPs) catalyse the dephosphorylation of phosphotyrosine residues and together with protein tyrosine kinases (PTKs) regulate the tyrosine phosphoproteome in cells. ${ }^{1}$ Several PTPs have been identified as attractive therapeutic targets in human diseases such as cancer, diabetes, obesity, neurodegenerative disorders and inflammation. ${ }^{2-4}$

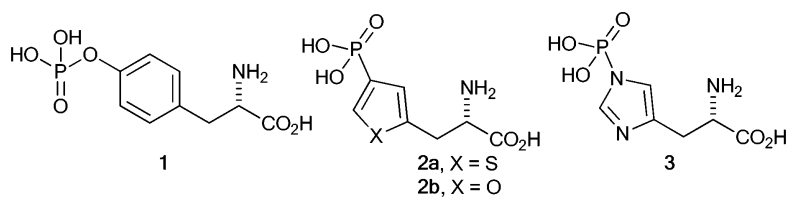

Since phosphotyrosine $\mathbf{1}$ is unstable under typical biological conditions, interest in the biological roles of PTPs concomitantly led to the development of stable analogues of phosphotyrosine., These analogues, azobenzene phosphonate (ABP), ${ }^{6}$ phosphotyramine, ${ }^{7}$ phosphonomethylphenylalanine (Pmp), ${ }^{8}$ and difluorophosphono-methylphenylalanine (F2Pmp $)^{9,10}$ have been invaluable tools in unraveling the importance of phosphotyrosine in cellular processes. These benzenoid analogues have been useful not only as immunogens to generate antibodies that recognise phosphotyrosine with high specificity without cross-reactivity to other phosphoamino acids, but also as inhibitors and affinity ligands for PTPs and other pTyr binding proteins.

${ }^{a}$ Department of Chemistry, The University of Sheffield, Dainton Building, Sheffield, S3 7HF, UK. E-mail: r.f.w.jackson@shef.ac.uk

${ }^{b}$ Department of Infection and Immunity, Academic Unit of Respiratory Medicine, The University of Sheffield Medical School, Beech Hill Road, Sheffield, S10 2TH, UK. E-mail: r.muimo@shef.ac.uk

$\dagger$ Electronic supplementary information (ESI) available: Experimental procedures and NMR spectra for $2 \mathbf{a}, \mathbf{4 , 5}, \mathbf{7}$ and $\mathbf{8}$. See DOI: $10.1039 / \mathrm{c} 4 \mathrm{cc} 03393 \mathrm{k}$
Significantly, incorporation of Pmp and F2Pmp into peptide sequences, has provided phospho-site specific antibodies and more potent or specific phosphatase inhibitors. These peptides have also been used as affinity ligands and in structural studies where they have provided invaluable information about proteinprotein interactions. ${ }^{8-11}$ A major limitation is the absence of specific inhibitors against many PTPs. Consequently, PTP research has lagged behind PTK studies and the related field of serine/ threonine protein phosphatases. ${ }^{12,13}$

Motivated by a report on the corresponding furan $\mathbf{2 b}$ that suggested it might be a non-hydrolysable and non-isomerisable analogue of phosphohistidine $3,{ }^{14}$ we synthesised 4-phosphothiophen-2-yl alanine, 2a, with the aim of establishing whether the thiophene would better mimic the imidazole in 3 or the benzene ring in 1 . Hirao cross coupling ${ }^{15}$ between diethyl phosphite and 3-bromothiophene gave 3-diethoxylphosphothiophene 4. Iodination of 4 gave, in addition to the 2,5-diododerivative $(18 \%)$, the iodothiophene 5 , which was subjected to Negishi cross-coupling with zinc reagent 6 using our recently optimised conditions ${ }^{16}$ to give the protected amino acid 7 . Sequential deprotection then gave 4-phosphothiophen-2-yl alanine 2a, via the corresponding acid $\mathbf{8}$ (Scheme 1).

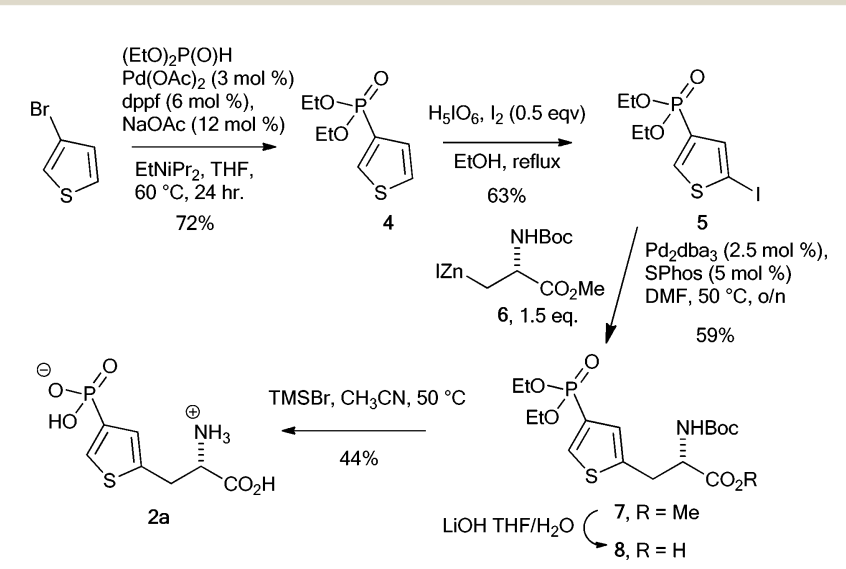

Scheme 1 Synthesis of 4-phosphothiophen-2-yl alanine $\mathbf{2 a}$. 
To explore its potential as a phosphoamino acid analogue, compound 2a was used as a hapten to produce antibodies. Polyclonal antibodies were raised in rats using KLH-conjugated analogue 2a as the immunogen. In one of the initial reports of a phosphotyrosine antibody generated using ABP as immunogen, Frackelton et al. observed cross-reactivity towards phosphohistidine $3 .^{6}$ In the course of this study, several non-hydrolysable analogues of phosphohistidine have been reported including phosphopyrrole, ${ }^{17}$ phosphoryltriazolylalanine isomers, ${ }^{18,19}$ a sulfonamide-based transition-state analogue of enzymatic phosphohistidine dephosphorylation ${ }^{20}$ and phosphoryltriazolylethylamine. ${ }^{21}$ Use of phosphoryltriazolylalanine as a mimic of one phosphohistidine in a histone $\mathrm{H} 4$ peptide allowed the generation of antibodies that did not universally recognise phosphohistidine in proteins. $^{18}$ Antibodies subsequently generated to phosphoryltriazolylethylamine showed cross-reactivity towards phosphohistidine and phosphotyrosine. ${ }^{21}$ The reactivity of the antibodies generated to $2 \mathbf{a}$ was initially tested using dot-blots spotted with BSA-conjugated histidine, phosphoserine, phosphothreonine, phosphotyrosine and $\tau$-phosphohistidine. Antibodies showed strong reactivity towards phosphotyrosine without any cross-reactivity for $\tau$-phosphohistidine, histidine, tyrosine or other phosphohydroxy amino acids (results not shown). This suggested that 2a was a potential analogue for phosphotyrosine. The antibodies were therefore rescreened against tyrosine, phosphotyrosine, phosphoserine, phosphothreonine and $\tau$-phosphohistidine (Fig. 1, entry A). The antibodies showed high selectivity for phosphotyrosine with minimal side reactivity towards BSA-conjugated $\tau$-phosphohistidine (Fig. 1, A lane v). However, this apparent $\tau$-phosphohistidine crossreactivity was simply removed by affinity depletion using a histidine-KLH glutaraldehyde conjugated Sepharose column (Fig. 1, entry B) suggesting that the cross linker itself produced a reasonable immune response.

The thiophene in analogue $\mathbf{2 a}$ is evidently a poor mimic of the corresponding imidazole present in $\tau$-phosphohistidine, but a good mimic of the benzene ring present in phosphotyrosine, which has resulted in a polyclonal serum that detects phosphotyrosine in preference to $\tau$-phosphohistidine.

Competition studies, performed by ELISA on the purified polyclonal antibody, showed no binding or inhibition of the antisera by any of the amino acids apart from phosphotyrosine (Fig. 2). This indicates that while analogue 2a may not be a
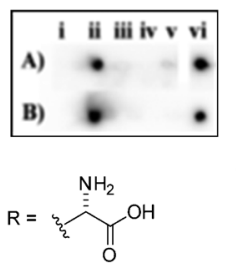

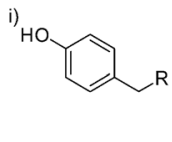

ii)

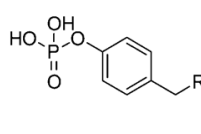

iv)

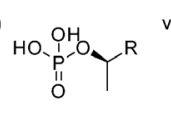

)
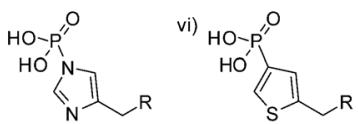

Fig. 1 Dot-blots of 4-phosphothiophen-2-yl alanine generated antibody using (A) crude polyclonal antibody and (B) polyclonal antibody affinity depleted with Sepharose bound/glutaraldehyde linked histidine-KLH when probed for (i) tyrosine, (ii) phosphotyrosine, (iii) phosphoserine, (iv) phosphothreonine, (v) $\tau$-phosphohistidine, (vi) 4-phosphothiophen-2-yl alanine.

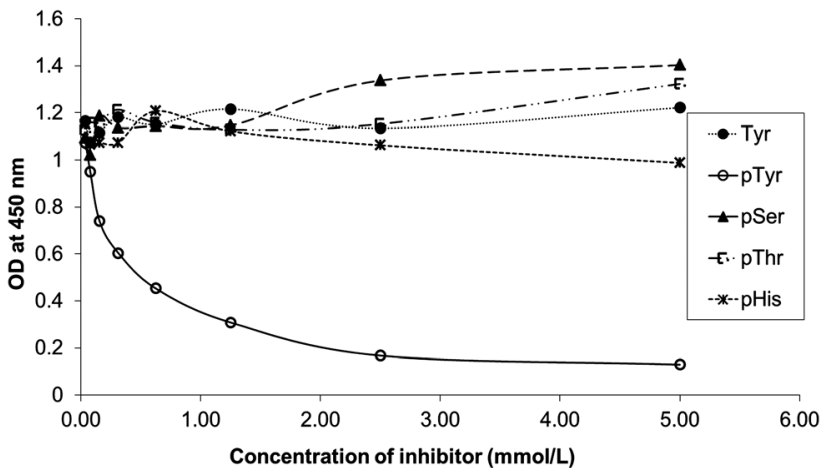

Fig. 2 Competitive inhibition ELISA study using anti-sera generated from 4-phosphothiophen-2-yl alanine, $2 \mathbf{a}$.

suitable analogue for $\tau$-phosphohistidine it was likely to be a useful analogue for phosphotyrosine. Competitive inhibition ELISA analysis using $O$-phospho--tyrosine (pTyr) or 2a, demonstrated that the antibody generated from $2 \mathbf{a}$ has a higher affinity towards 2a compared to phosphotyrosine (Fig. 3, see inset), as expected.

In Western blot analysis, using cytosolic fractions obtained from sheep tracheal epithelia, the antibody generated from $\mathbf{2 a}$ gave a staining profile comparable to that of a commercially available anti-phosphotyrosine monoclonal antibody (pY99) albeit with varying degrees of staining or detection intensity for various protein bands by either antibody (Fig. 4A). This indicates that the antibody generated by $\mathbf{2} \mathbf{a}$ is able to detect phosphotyrosine containing proteins and recognises many protein bands that are also detected by the anti-phosphotyrosine pY99. Interestingly, the antibody generated from $2 \mathrm{a}$ also detected proteins that were not detected by the pY99 (and vice versa). This is consistent with previous studies where different types of antibodies provided varied results in the same systems. ${ }^{22}$

Furthermore, in total lysates of human bronchial epithelial cell lines (16HBE14o-) treated with $\mathrm{Na}_{3} \mathrm{VO}_{4}$ (a broad spectrum PTP inhibitor) for $45 \mathrm{~min}$, both the antibody generated from 2a and pY99 detect enhanced phosphorylation of a high molecular

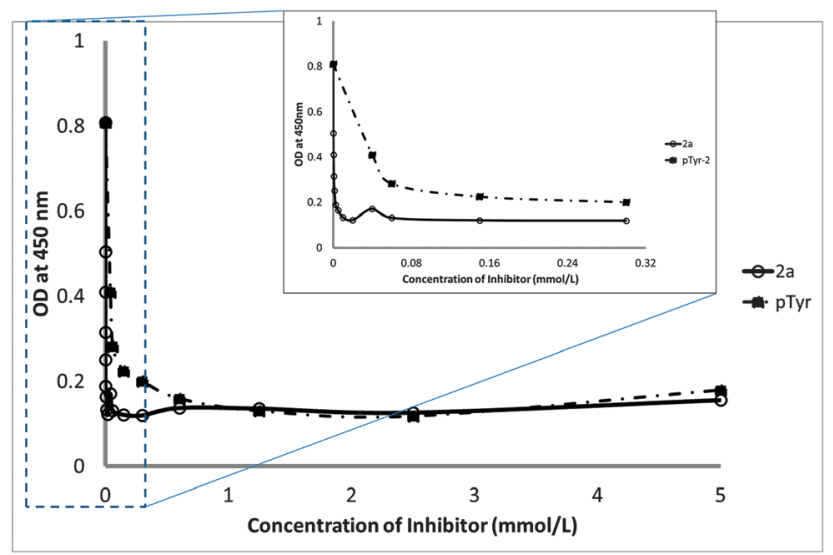

Fig. 3 A competitive inhibition ELISA study involving O-phospho-Ltyrosine (pTyr) and 4-phosphothiophen-2-yl alanine, $2 a$ using anti-sera generated from analogue, $\mathbf{2 a}$. 
A

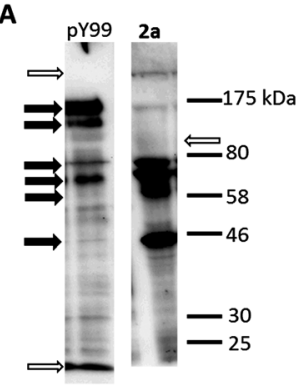

B

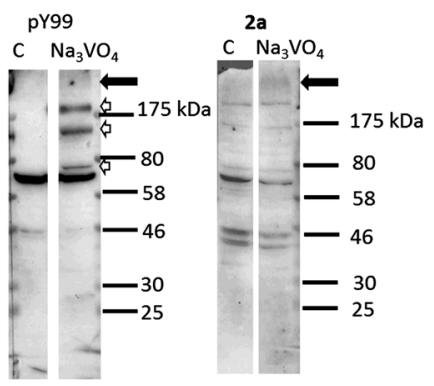

C

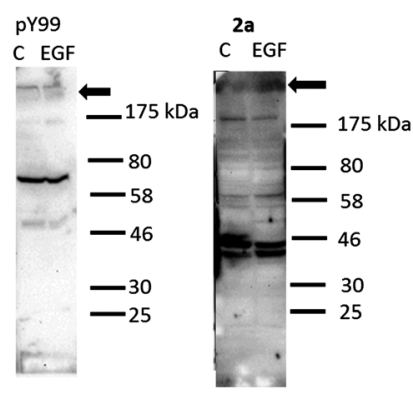

Fig. 4 Comparison of antibody generated to $\mathbf{2 a}$ and commercially available anti-phosphotyrosine monoclonal antibody (pY99). (A) Western blots of cytosolic fraction from sheep tracheal epithelia probed with pY99 or 2a antibody. Arrows highlight similarities (filled) or differences (unfilled). Western blots of lysates of human bronchial epithelial cell lines (16HBE14O-) treated $\pm \mathrm{Na}_{3} \mathrm{VO}_{4}(10 \mathrm{mM})$ for $45 \mathrm{~min}(\mathrm{~B})$ or $\pm \mathrm{EGF}(1 \mathrm{nM})$ for 4 hours $(\mathrm{C})$ and probed with pY99 (left panel) or the antibody generated from 2a (right panel), ( $C=$ control, untreated). Arrows/arrowheads highlight similarities (filled) or differences (unfilled).

weight protein (Fig. 4B, filled arrow). However, pY99 additionally detects enhanced phosphorylation of three other proteins (approx. $75,100,180 \mathrm{kDa}$ ) in $\mathrm{Na}_{3} \mathrm{VO}_{4}$ treated cells (Fig. 4B, left panel, unfilled arrow heads). Interestingly, both antibodies also detect several other proteins that were insensitive to $\mathrm{Na}_{3} \mathrm{VO}_{4}$ and in this regard, pY99 antibodies detected two, whereas the antibody generated from $2 \mathbf{a}$ detected six, of these proteins. In lysates from cells stimulated with epidermal growth factor (EGF, $1 \mathrm{nM}$ for 4 hours), both the antibody generated from 2a and pY99 detected enhanced phosphorylation of a high molecular weight protein (Fig. 4C, filled arrow).

In conclusion, we have synthesized 4-phosphothiophen-2-yl alanine, $2 \mathbf{a}$ and demonstrated its potential as a novel 5-membered ring analogue of phosphotyrosine. Our data suggests that analogue 2a, and resulting polyclonal antibody, may potentially be a useful tool to both characterize the biological effects of phosphotyrosine and the role of specific PTP(s) in cells.

We thank the EPSRC Life Sciences Interface DTC (ML). The 16HBE140- cells were provided by Dr D. Gruenert (California Pacific Medical Center, CA).

\section{Notes and references}

1 T. Hunter, Cell, 2000, 100, 113-127.

2 J. A. Ardura and P. A. Friedman, Pharmacol. Rev., 2011, 63, 882-900.

3 L. Bialy and H. Waldmann, Angew. Chem., Int. Ed., 2005, 44, 3814-3839.

4 A. Noeren-Mueller, I. Reis-Correa, Jr., H. Prinz, C. Rosenbaum, K. Saxena, H. J. Schwalbe, D. Vestweber, G. Cagna, S. Schunk, O. Schwarz, H. Schiewe and H. Waldmann, Proc. Natl. Acad. Sci. U. S. A., 2006, 103, 10606-10611.

5 K. Shen, Methods, 2007, 42, 234-242.

6 A. R. Frackelton, A. H. Ross and H. N. Eisen, Mol. Cell. Biol., 1983, 3, 1343-1352.

7 G. Steinhilber, M. W. Sproll, J. M. Wolff and F. A. Anderer, Anticancer Res., 1990, 10, 907-911.

8 Z. Y. Zhang, D. Maclean, D. J. McNamara, T. K. Sawyer and J. E. Dixon, Biochemistry, 1994, 33, 2285-2290.

9 M. R. Groves, Z. J. Yao, P. P. Roller, T. R. Burke and D. Barford, Biochemistry, 1998, 37, 17773-17783.

10 M. Akamatsu, P. P. Roller, L. Chen, Z. Y. Zhang, B. Ye and T. R. Burke, Bioorg. Med. Chem., 1997, 5, 157-163.

11 K. Shen, L. X. Qi and L. Stiff, Curr. Pharm. Des., 2010, 16, 3101-3117.

12 J. J. Fernandez, M. L. Candenas, M. L. Souto, M. M. Trujillo and M. Norte, Curr. Med. Chem., 2002, 9, 229-262.

13 C. F. B. Holmes, J. T. Maynes, K. R. Perreault, J. F. Dawson and M. N. G. James, Curr. Med. Chem., 2002, 9, 1981-1989.

14 C. Schenkels, B. Erni and J. L. Reymond, Bioorg. Med. Chem. Lett., 1999, 9, 1443-1446.

15 M. Kalek, M. Jezowska and J. Stawinski, Adv. Synth. Catal., 2009, 351, 3207-3216.

16 A. J. Ross, H. L. Lang and R. F. W. Jackson, J. Org. Chem., 2010, 75, 245-248.

17 P. V. Attwood, M. J. Piggott, X. L. Zu and P. G. Besant, Amino Acids, 2007, 32, 145-156.

18 J.-M. Kee, B. Villani, L. R. Carpenter and T. W. Muir, J. Am. Chem. Soc., 2010, 132, 14327-14329.

19 T. E. McAllister, M. G. Nix and M. E. Webb, Chem. Commun., 2011, 47, 1297-1299.

20 M. F. Eerland and C. Hedberg, J. Org. Chem., 2012, 77, 2047-2052.

21 J. M. Kee, R. C. Oslund, D. H. Perlman and T. W. Muir, Nat. Chem. Biol., 2013, 9, U416-U428.

22 J. Y. J. Wang, Anal. Biochem., 1988, 172, 1-7. 